\title{
La oxidación de componentes grasos y formación de hexanal en la curación del salchichón
}

\author{
Por J. Chasco, M.J. Beriain y J. Bello \\ Departamento de Bromatologia. Facultad de Farmacia. \\ Universidad de Navarra. 31008. PAMPLONA (ESPAÑA)
}

\section{RESUMEN}

La oxidación de componentes grasos y formación de hexanal en la curación del salchichón

Se ha estudiado la formación de alcanales de cadena corta (C3-C6) durante el proceso de curación de un salchichón estándar elaborado en planta piloto. Se ha comparado sus contenidos finales con los obtenidos para otros tres lotes de salchichón de fabricación industrial (I, II, III). Con excepción del hexanal, que se encuentra en cantidades importantes, los otros tres aldehídos aparecen en cantidades muy reducidas o incluso no se detectan. No se ha obtenido una correlación estadística entre el contenido en hexanal y los niveles de oxidación de los componentes grasos valorados a través del indice de peróxidos, el número T.B.A. o los compuestos carbonilos totales.

Sin embargo, si existe una cierta correlación entre el contenido en hexanal y las relaciones oleico/linoleico de cada lote de salchichón. La ausencia de hexanal en todos los salchichones del lote II viene correspondida por una mayor proporción de ácido linoleico entre sus AGL. Se propone la determinación de hexanal como un modo útil de comprobar si el mecanismo oxidativo de las grasas durante el proceso de curación ha sido correcto.

PALABRAS-CLAVE: Hexanal - Oxidación lipídica - Proceso de curación - Salchichón.

\section{SUMMARY}

Lipidic components oxidation and hexanal formation during the curing of saucisson

It has studied formation of alcanals of low weight (C3-C6) during the curing of standard Saucisson manufactured in pilot plant. Aldehydes content have been compared to others obtained in three batches of industrial process (I, II y III). Except to hexanal, present in important amounts, the rest of aldehydes appear in reduced levels even are not detected. Statistical correlation has not been obtained between hexanal content and oxidation levels of lipidic components titrated by peroxides value, T.B.A. number or total carbonyl compounds. However, certain correlation has been found between hexanal content and ratio of oleic/linoleic of each batch of saucisson. Absence of hexanal in all of saucissons of batch II is corresponded by a greater proportion of linoleic acid between their A.G.L. It is proposed determination of hexanal like an useful method to check whether oxidative mechanism of lipids during the curing has been corrected.

KEY-WORDS: Curing process - Hexanal - Lipidic oxidation Saucisson.

\section{INTRODUCCION}

Los componentes grasos de los embutidos crudoscurados suelen presentar fenómenos de degradación/oxi- dación en su proceso de maduración-desecación, dando lugar a un número importante de sustancias volátiles entre ellas aldehídos (Bailey et al., 1980). Este conjunto de reacciones en el que toman parte los lípidos desempeña un papel importante en el desarrollo del aroma típico de cada embutido (Shahidi, 1989), a través de mecanismos todavía no bien comprendidos (Gray et al., 1984). Una de las reacciones que se considera más relevante en este proceso es la oxidación del ácido linoleico, que lleva a la formación de hexanal (Ellis et al., 1968) (Henderson et al., 1980) (Frankel et al., 1981). La valoración del contenido en este aldehído se ha propuesto como una fórmula válida para predecir la calidad de algunos aceites vegetales (Dupuy et al., 1976) (Warner et al., 1978). Dado los niveles de ácido linoleico que participa en la composición de las grasas de cerdo que toman parte en la pasta embutida del salchichón, cabe pensar en el hexanal como uno de los principales aldehídos volátiles formados en los procesos oxidativos implicados en la curación de este embutido. Su valoración podría ser útil como un método de control de calidad aplicable a su fabricación industrial.

Con el presente trabajo se ha querido profundizar en las posibles relaciones que puedan existir entre la formación de aldehídos volátiles de cadena corta (propanal, butanal, pentanal, hexanal) y la oxidación de los componentes grasos del salchichón. Para ello se han determinado los niveles de estos aldehídos a lo largo del proceso de curación de un salchichón estándar comparándolos con los obtenidos para otros productos acabados de fabricación industrial. También se han analizado parámetros reconocidos como índices de valoración del nivel de oxidación de los triglicéridos (índice de peróxidos, número T.B.A., compuestos carbonilos totales), junto a los contenidos en ácidos grasos totales y libres de la pasta del salchichón.

\section{PARTE EXPERIMENTAL}

Para la realización del presente trabajo se ha sometido a estudio cuatro lotes de salchichones, de tres piezas cada uno, de los cuales tres correspondian a fabricación 
industrial (I, II y III) y uno a un embutido estándar elaborado en planta piloto. Su elaboración se hizo con arreglo a la formulación siguiente: magro de cerdo $(60 \%)$, carne de vacuno $(20 \%)$, tocino $(20 \%)$, sal común $(20 \mathrm{~g} / \mathrm{Kg})$, azúcares $(15 \mathrm{~g} / \mathrm{Kg})$, pimienta negra $(4 \mathrm{~g} / \mathrm{Kg})$, polifosfatos (2 g/Kg), ascorbato sódico $(0,3 \mathrm{~g} / \mathrm{Kg})$, nitritos $(0,2 \mathrm{~g} / \mathrm{Kg})$ y nitratos $(0,1 \mathrm{~g} / \mathrm{Kg})$.

El magro de cerdo, la carne de vacuno y el tocino se trocean por separado en una máquina cortadora. Se mezclan y se pasan a una máquina picadora que lleva placa de $3 \mathrm{~mm}$. Se le añade el resto de los ingredientes y se amasa con vacío para eliminar el aire ocluído. Finalmente se embute en tripa de $60 \mathrm{~mm}$ de diámetro. Todo el proceso se lleva a cabo a la temperatura de $4^{\circ} \mathrm{C}$. Las piezas embutidas se mantienen durante tres días en cámara-estufa por la que circula aire a $25^{\circ} \mathrm{C}$ con una humedad relativa (HR) del $90 \%$. A continuación se sigue el proceso en cámara-secadero donde las piezas permanecen a $15-18^{\circ} \mathrm{C}$ y $75-80$ de HR durante cuatro semanas, momento en el que el salchichón estándar se considera apto para su consumo.

Para la preparación de las muestras se toman las piezas enteras, se elimina la envoltura y se trocea en toda su magnitud. Se toman porciones alternas y se vuelve a mezclar. Estas operaciones se llevan a cabo a $2^{\circ} \mathrm{C}$ y se repiten tantas veces como sea necesario para que resulte una mezcla homogénea, representativa de todo el conjunto. De cada pieza preparada se tomaron tres muestras para realizar las determinaciones de cada parámetro analizado.

Los datos recogidos en las tablas representan los valores medios de tres piezas (nueve determinaciones en total) con sus correspondientes errores estándars.

Para el estudio del desarrollo de aldehídos volátiles en el salchichón estándar se hicieron determinaciones en diferentes fases del proceso de curación: a) amasado, b) estufado, c) 7 días secadero, d) 14 días secadero, e) 21 días secadero y f) 28 días secadero (producto acabado).

Extractor de la Casa Tecator. Los cloruros se valoraron por el método de Volhard. Para la medición del pH se usó el aparato ORION RESEARCH microprocessor ionalyzer con electrodo selectivo y para la Actividad de Agua se empleó aparato EEJA-3 NOVASINA.

El análisis de los aldehídos volátiles se llevó a cabo en un equipo Perkin-Elmer modelo Sigma-300/Dual FID Chromatograph con programador Sigma-15/Chromatograph/Data Station y aplicador de muestras de espacio de cabeza Perkin-Elmer HS-6. Se usó una columna de acero inox. 1,8 $\mathrm{m} \times 1 / 8$ " con Chromosorb W-AW 80/100, que lleva Carbowax 1.500 al $15 \%$. Se fijaron las siguientes condiciones de trabajo:

- Condiciones del control HS-6: Gas portador inerte, nitrógeno. Temperatura de las muestras encapsuladas en viales, $70^{\circ} \mathrm{C}$. Presurización (tiempo de inyección del N2 en vial), 1 minuto.

- Condiciones del cromatógrafo de gases: Temperatura del horno, $90^{\circ} \mathrm{C}$. Temperatura del detector, $240^{\circ} \mathrm{C}$. Temperatura del inyector, $200^{\circ} \mathrm{C}$.

Se usaron patrones de propanal, butanal, pentanal y hexanal de Polyscience Corporation Analytical Standars.

Para caracterizar las modificaciones de las grasas se hicieron las siguientes determinaciones: el índice de peróxidos (UNE 55-023-73, 1973), el número T.B.A. (Tarladgis et al.,1960), los compuestos carbonilos (Henick et al., 1954). Para la identificación y cuantificación de los ácidos grasos totales (AGT) y libres (AGL) se ha usado el equipo PERKIN-ELMER, modelo Sigma 300 de acuerdo con (UNE 55-118-79, 1979).

\section{Análisis de datos}

Los resultados obtenidos se han sometido a un análisis multivariante de componentes principales, así como al análisis de la varianza y test de comparaciones múltiples de $t$ de Tukey para cada variable. Para todo ello se ha utilizado el programa DATA DESK, versión Velleman y Velleman (1988) de Odesta Corporation, con un ordenador Macintosh SE 1/20 Apple Computer.

\section{RESULTADOS Y DISCUSION}

En primer lugar se han determinado los parámetros de tipo general que suelen estar implicados, o son consecuencia, del proceso de maduración-desecación que experimenta el salchichón durante su tecnología de fabricación: contenido acuoso, proteína total, grasa total, cloruros, $\mathrm{pH}$ y actividad de agua (Tabla I).

Tabla I

Parámetros generales implicados en el proceso de maduración-desecación del Salchichón

\begin{tabular}{ccccccc}
\hline Muestra & $\begin{array}{c}\text { Contenido } \\
\text { acuoso } \\
\%\end{array}$ & $\begin{array}{c}\text { Proteina } \\
\text { total } \\
\%\end{array}$ & $\begin{array}{c}\text { Grasa } \\
\text { total } \\
\%\end{array}$ & $\begin{array}{c}\text { Cloruros } \\
\% \text { CINa }\end{array}$ & pH & $\begin{array}{c}\text { Actividad de } \\
\text { Agua }\end{array}$ \\
\hline Estándar & $30,9 \pm 0,5$ & $22,3 \pm 0,2$ & $36,3 \pm 0,3$ & $4,66 \pm 0,02$ & $4,85 \pm 0,03$ & $0,819 \pm 0,001$ \\
I & $30,0 \pm 0,6$ & $22,1 \pm 0,3$ & $38,5 \pm 0,4$ & $4,69 \pm 0,02$ & $4,80 \pm 0,04$ & $0,813 \pm 0,001$ \\
II & $28,0 \pm 0,5$ & $22,0 \pm 0,2$ & $37,1 \pm 0,7$ & $4,64 \pm 0,02$ & $4,79 \pm 0,04$ & $0,806 \pm 0,001$ \\
III & $27,6 \pm 0,7$ & $21,5 \pm 0,5$ & $36,9 \pm 0,6$ & $4,73 \pm 0,03$ & $4,72 \pm 0,02$ & $0,802 \pm 0,001$ \\
\hline
\end{tabular}


Los valores de $\mathrm{pH}$ y actividad de agua encontrados en cada una de las piezas de los cuatro lotes de salchichones estudiados se pueden considerar normales para este tipo de embutido y pone de manifiesto que todos ellos han recibido un proceso de curación correcto (Astiasaran et al., 1990).
El desarrollo del contenido en aldehídos volátiles (propanal, butanal, pentanal y hexanal) en las distintas etapas del proceso de curación del salchichón estándar se refleja en la Tabla II. Y el contenido de estos aldehídos encontrados en las distintas muestras de salchichones estudiados se reúnen en la Tabla III.

Tabla II

Desarrollo de Aldehídos volátiles ( $\mu \mathrm{g} / \mathrm{g}$ de muestra seca) con el proceso de curación del Salchichón estándar elaborado en planta piloto

\begin{tabular}{|c|c|c|c|c|c|c|c|}
\hline $\begin{array}{l}\text { Aldehído } \\
\text { volátil }\end{array}$ & Amasado & Estufado & $\begin{array}{c}\text { Secado } \\
\text { 1. a semana }\end{array}$ & $\begin{array}{l}\text { Secado } \\
\text { 2.. }{ }^{\mathrm{a}} \text { semana }\end{array}$ & $\begin{array}{c}\text { Secado } \\
\text { 3. }{ }^{\text {a }} \text { semana }\end{array}$ & $\begin{array}{c}\text { Secado } \\
\text { 4. }{ }^{\mathrm{a}} \text { semana }\end{array}$ & Significación \\
\hline Propanal & - & - & - & - & - & - & \\
\hline Butanal & - & - & - & - & - & - & \\
\hline Pentanal & - & - & - & - & $0,59 \pm 0,01 a$ & $0,62 \pm 0,05^{a}$ & NS \\
\hline Hexanal & - & - & $49,17 \pm 0,86$ & $53,80 \pm 0,47$ & $60,57 \pm 3,33$ & $66,11 \pm 2,86$ & $* * *$ \\
\hline
\end{tabular}

Valores con el mismo superindice son estadisticamente semejantes $(p>0,05)$

**, $p<0,001$

NS, diferencias no significativas $(p>0,05)$.

Tabla III

Contenido en Aldehídos volátiles ( $\mu \mathrm{g} / \mathrm{g}$ de muestra seca) en muestras de Salchichón

\begin{tabular}{cccccc}
\hline $\begin{array}{c}\text { Aldehido } \\
\text { volátil }\end{array}$ & Estándar & I & II & III & Significación \\
\hline Propanal & - & $0,29 \pm 0,01$ & - & $0,25 \pm 0,01$ & ${ }^{* *}$ \\
Butanal & - & $0,22 \pm 0,01$ & $0,45 \pm 0,02$ & $0,14 \pm 0,00$ & ${ }^{* * *}$ \\
Pentanal & $0,62 \pm 0,05^{\mathrm{a}}$ & $0,32 \pm 0,02$ & $0,64 \pm 0,07 \mathrm{a}$ & $0,50 \pm 0,02$ & ${ }^{* * *}$ \\
Hexanal & $66,11 \pm 2,86 \mathrm{a}$ & $64,76 \pm 0,76^{\mathrm{a}}$ & vestigios & $65,59 \pm 0,92^{\mathrm{a}}$ & $\mathrm{NS}$ \\
\hline
\end{tabular}

Valores con el mismo superíndice son estadísticamente semejantes $(p>0,05)$

$*$, $p<0,01$.

$\cdots, p<0,001$

Durante la curación del salchichón estándar no se ha detectado en ningún momento propanal ni butanal. El pentanal aparece a la tercera semana de permanecer en secadero, aunque en cantidades muy reducidas $(0,6)$. En cambio, el hexanal se detecta ya al final de la primera semana de curación en cantidades importantes $(49,2)$, que se incrementan con la maduración-desecación para alcanzar valores de 66,1 en el producto acabado. Estos niveles concuerdan con los encontrados para los salchichones de los otros lotes de fabricación industrial, con la excepción del lote II en los que el hexanal sólo se detecta a niveles de vestigios.
Se sabe que el hexanal es un aldehído predominante entre los compuestos de ruptura de la peroxidación lipídica de los ácidos grasos w6 presentes en los productos cárnicos (Persson et al., 1973) (Mcleod et al., 1976), aunque en los derivados curados venga a ser solamente un $7 \%$ del que se forma en los derivados cocidos (Shahidi, 1989).

El nivel de oxidación presentado por la grasa de estas muestras de salchichón se ha determinado mediante valoración del índice de peróxidos, número T.B.A. y compuestos carbonilos totales (Tabla IV). 
Tabla IV

Indices de oxidación de la grasa en muestras de Salchichón

\begin{tabular}{|c|c|c|c|c|c|}
\hline Parámetro & Estándar & I & II & III & Significación \\
\hline $\begin{array}{l}\text { Indice de } \\
\text { Peróxidos } \\
\text { meq 02/Kg }\end{array}$ & $9,38 \pm 0,43^{a}$ & $12,78 \pm 0,85$ & $9,59 \pm 0,66^{a}$ & $13,49 \pm 0,99$ & $\star \star \star \star$ \\
\hline $\begin{array}{l}\text { n. }{ }^{9} \text { T.B.A. } \\
\text { mg MA/g }\end{array}$ & $0,55 \pm 0,03^{a}$ & $0,56 \pm 0,05^{a}$ & $0,51 \pm 0,04^{a}$ & $0,54 \pm 0,05^{a}$ & NS \\
\hline $\begin{array}{l}\text { Indice de } \\
\text { carbonilos } \\
\mu \mathrm{m} C O / g\end{array}$ & $14,70 \pm 0,41^{a}$ & $21,14 \pm 0,35$ & $15,56 \pm 0,24^{a}$ & $25,45 \pm 0,27$ & $\star * *$ \\
\hline
\end{tabular}

Valores con el mismo superindice son estadisticamente semejantes $(p<0,05)$.

$\cdots, p<0,001$.

NS, diferencias no significativas $(p>0,05)$.

Entre los ácidos grasos insaturados precursores de hexanal destaca el ácido linoleico (Ellis et al., 1968) (Henderson et al., 1980) (Frankel et al., 1981), principalmente a través de la oxidación a 13-monohidroperóxidos, aunque también se pueda formar de los 9monohidroperóxidos, pero en cantidades más reducidas (Chan et al., 1976). La oxidación del ácido oleico puede originar porcentajes muy pequeños de alcanales volátiles C2-C6, porque este ácido insaturado suele degradarse con preferencia en aldehídos insaturados, a través de una beta-escisión de sus hidroperóxidos (Loury, 1972). También una pequeña cantidad de propanal se puede formar en la oxidación del ácido linolénico y sus homólogos w3 (Grosch, 1987). Posiblemente, todas estas circunstancias expliquen las cantidades tan reducidas, o inexistentes, de propanal, butanal y pentanal encontradas en todas las piezas de salchichón que han sido analizadas.

Algunos autores han señalado una correlación entre el contenido en hexanal de los productos cárnicos cocidos y la cantidad de sustancias reactivas con el ácido 2tiobarbitúrico o número T.B.A. (Shahidi et al., 1987). En nuestro estudio no se ha encontrado correlación entre los valores de hexanal en los salchichones analizados y los anteriores índices de oxidación de la grasa. Desde luego se admite que a la temperatura ambiente, propia del proceso de curación de este embutido, se suele obtener una escasa proporción de compuestos volátiles en comparación con la cantidad primaria de monohidroperóxidos que se forman (Grosch, 1987). Pero también se admite la posibilidad de que los volátiles se puedan desarrollar por un mecanismo diferente a la degradación de monohidroperóxidos en los productos cárnicos curados (Shahidi et al., 1987), e incluso se ha hablado de peróxidos polares muy lábiles, aunque no se haya podido aclarar su estructura (Morita et al., 1973) (Morita et al., 1976).

El índice de peróxidos se considera aceptable para detectar la oxidación de los lípidos presentes en los productos cárnicos, pero no adecuado para establecer una correlación entre sus valores y el nivel de alteración de la grasa (Melton, 1983). Los cuatro lotes de salchichones estudiados presentan diferencias altamente significativas para la oxidación de sus lípidos valorada según el índice de peróxidos o la cantidad de compuestos carbonilos totales, pero en cambio resultan significativamente semejantes cuando se comparan los números T.B.A.

Las mayores diferencias entre los valores medios de los cuatro lotes se observan para los compuestos carbonilos, tal vez explicable por el comportamiento de este tipo de compuestos, capaces de presentar oscilaciones a lo largo de la curación de los embutidos (Lizarraga et al., 1989), debido a su doble origen: la fermentación de los azúcares al inicio (de Ketelaere et al., 1974) y el metabolismo de los peróxidos lipídicos, por parte de los microorganismos, al final (Alford et al., 1971).

- Sorprende que todos los salchichones analizados correspondientes al lote II presenten solamente vestigios de hexanal, lo que hace pensar en algún agente inhibidor de la formación de este aldehído a partir del ácido linoleico. Recientemente, se ha señalado un efecto sinérgico del ácido ascórbico y tripolifosfato sódico, que juntos y a ciertos niveles provocan una reducción del contenido en hexanal de los productos cárnicos (UNE 55-023-73, 1973) (Yun et al., 1987). Estos suelen ser dos productos incluidos en la formulación industrial de los salchichones españoles y en el caso del lote II podrían haber alcanzado las proporciones adecuadas para poner de manifiesto su actividad sinérgica.

La formación de aldehídos volátiles como consecuencia de las oxidaciones que tienen lugar en los ácidos grasos insaturados debe tener su reflejo en la composición de los ácidos grasos que forman parte de los triglicéridos, pero sobre todo en los porcentajes relativos de los ácidos grasos libres.

El porcentaje relativo obtenido para los distintos ácidos grasos totales (AGT) se recogen en la Tabla $V$ mientras que los porcentajes de los ácidos grasos libres ( $A G L$ ) se reúnen en la Tabla VI. Tanto los AGT como los AGL presentan relaciones de saturación/insaturación características de cada lote de salchichón estudiado. En la Tabla VII se expresan los resultados obtenidos para las relaciones de los ácidos palmítico/palmitoleico, de los ácidos esteárico/oleico + linoleico + linolénico y de los dos ácidos oleico/linoleico. 
Tabla V

Acidos grasos totales (\% relativo) que integran la grasa en diferentes muestras de Salchichón

\begin{tabular}{|c|c|c|c|c|c|}
\hline Acido graso & Estándar & 1 & II & III & Significación \\
\hline $\begin{array}{l}\text { Mirístico } \\
14: 0\end{array}$ & $1,55 \pm 0,14^{a}$ & $1,62 \pm 0,08 a$ & $1,96 \pm 0,20^{a}$ & $1,43 \pm 0,06^{a}$ & NS \\
\hline $\begin{array}{l}\text { Palmítico } \\
\text { 16:0 }\end{array}$ & $23,03 \pm 0,25 a$ & $23,20 \pm 0,26 a$ & $24,34 \pm 0,75$ & $22,05 \pm 0,03$ & * \\
\hline $\begin{array}{l}\text { Palmitoleico } \\
16: 1\end{array}$ & $5,08 \pm 0,16 a$ & $5,04 \pm 0,08 a$ & $5,18 \pm 0,32^{a}$ & $4,14 \pm 0,17$ & * \\
\hline $\begin{array}{l}\text { Esteárico } \\
18: 0\end{array}$ & $9,53 \pm 0,12^{a}$ & $9,16 \pm 0,10^{a}$ & $9,57 \pm 0,29 a$ & $9,69 \pm 0,27 a$ & NS \\
\hline $\begin{array}{l}\text { Oleico } \\
18: 1\end{array}$ & $45,98 \pm 0,20^{a}$ & $46,11 \pm 0,14$ & $44,36 \pm 0,62^{a}$ & $45,34 \pm 0,14^{a}$ & $\star$ \\
\hline $\begin{array}{l}\text { Linoleico } \\
18: 2\end{array}$ & $12,72 \pm 0,12^{a}$ & $13,04 \pm 0,12^{a}$ & $12,50 \pm 0,50 a$ & $13,13 \pm 0,20^{a}$ & NS \\
\hline $\begin{array}{l}\text { Linolénico } \\
18: 3\end{array}$ & $0,73 \pm 0,08$ & $0,60 \pm 0,04^{a}$ & $0,63 \pm 0,09 a$ & $0,85 \pm 0,05$ & ** \\
\hline
\end{tabular}

Valores con el mismo superíndice son estadísticamente semejantes $(p<0,05)$.

$\because p<0,05 ;{ }^{* *}, p<0,01$.

NS, diferencias no significativas $(p>0,05)$

Tabla VI

Acidos grasos libres (\% relativo) presentes en la masa de diferentes muestras de Salchichón

\begin{tabular}{|c|c|c|c|c|c|}
\hline Acido graso & Estándar & 1 & II & III & Significación \\
\hline $\begin{array}{l}\text { Mirístico } \\
14: 0\end{array}$ & $1,79 \pm 0,21^{a}$ & $2,10 \pm 0,15$ & $1,58 \pm 0,02^{a}$ & $2,69 \pm 0,26$ & \#* \\
\hline $\begin{array}{l}\text { Palmítico } \\
\text { 16:0 }\end{array}$ & $23,94 \pm 0,04^{a}$ & $24,01 \pm 0,16^{a}$ & $23,20 \pm 0,15^{a}$ & $23,17 \pm 0,19 a$ & NS \\
\hline $\begin{array}{l}\text { Palmitoleico } \\
16: 1\end{array}$ & $4,82 \pm 0,09 a$ & $4,92 \pm 0,08 a$ & $4,38 \pm 0,08^{b}$ & $4,00 \pm 0,08^{b}$ & ** \\
\hline $\begin{array}{l}\text { Esteárico } \\
\text { 18:0 }\end{array}$ & $9,36 \pm 0,30^{a}$ & $9,09 \pm 0,22^{a}$ & $9,66 \pm 0,19 a$ & $9,75 \pm 0,05^{a}$ & NS \\
\hline $\begin{array}{l}\text { Oleico } \\
18: 1\end{array}$ & $45,58 \pm 0,19$ & $45,80 \pm 0,18^{a}$ & $44,88 \pm 0,05^{a}$ & $42,64 \pm 0,21 a$ & $\star \star$ \\
\hline $\begin{array}{l}\text { Linoleico } \\
18: 2\end{array}$ & $12,89 \pm 0,08 a$ & $13,31 \pm 0,02$ & $13,99 \pm 0,08$ & $12,82 \pm 0,04^{a}$ & ** \\
\hline $\begin{array}{l}\text { Linolénico } \\
18: 3\end{array}$ & $0,78 \pm 0,04^{a}$ & $0,64 \pm 0,04$ & $0,79 \pm 0,08 a$ & $1,39 \pm 0,03$ & ** \\
\hline
\end{tabular}

Valores con el mismo superindice son estadisticamente semejantes $(p<0,05)$.

$*, p<0,01$.

NS, diferencias no significativas $(p>0,05)$

Tabla VII

Relaciones de insaturación obtenidas de los porcentajes de AGT y AGL determinados en las muestras de Salchichón estudiadas

\begin{tabular}{lcccc}
\hline & Estándar & I & II & III \\
\hline AGT & & & & \\
C16:0/C16:1 & 4,53 & 4,60 & 4,70 & 5,32 \\
C18:0/C18:1+2+3 & 0,16 & 0,15 & 0,17 & 0,16 \\
C18:1/C18:2 & 3,61 & 3,54 & 3,54 & 3,45 \\
AGL & & & & 5,79 \\
C16:0/C16:1 & 4,97 & 4,88 & 5,30 & 0,17 \\
C18:0/C18:1+2+3 & 0,16 & 0,15 & 0,16 & 3,32 \\
\hline
\end{tabular}


Efectivamente, los AGT presentan porcentajes relativos de los ácidos individuales que apenas difieren significativamente entre los cuatro lotes de salchichón. Sin embargo, los resultados obtenidos para la formación de AGL ponen de manifiesto diferencias significativas $(p<0,001)$ entre sus proporciones relativas, especialmente para los ácidos insaturados. Incluso se pone en evidencia un mayor contenido en linoleico libre en los salchichones del lote II, que sólo contienen vestigios de hexanal.

$\mathrm{El}$ análisis multivariante de todos los parámetros analizados permite una representación gráfica (figura 1) de la distribución de todas las muestras de cada lote de salchichones en función de los dos primeros componentes principales.

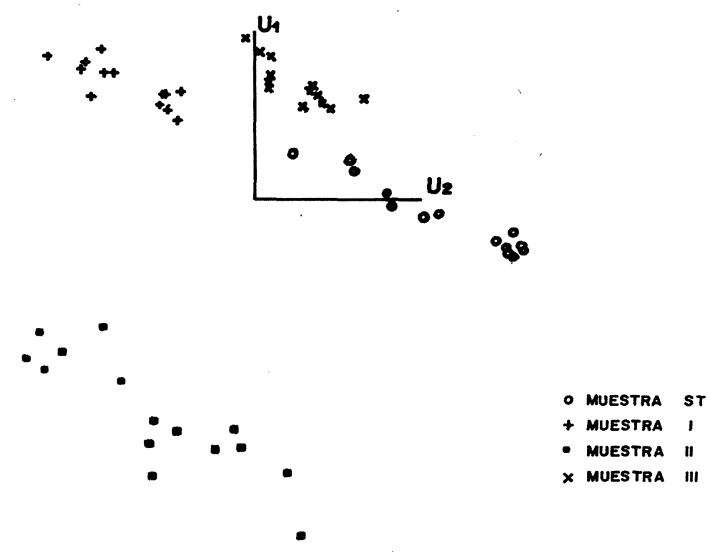

Figura 1

Representación gráfica de los diferentes tipos de salchichón sobre los dos primeros componenttes principales

Dicho análisis indica que los dos primeros componentes principales explican el $75 \%$ de la varianza. La representación gráfica (Fig. 1) sobre estos dos componentes principales resalta de una parte la mayor homogeneidad entre las piezas correspondientes a cada lote y de otra la evidente distinción del lote II, separado de los otros tres como consecuencia de su alteración en el mecanismo oxidativo de los lípidos que lleva a la formación de hexanal.

En conclusión, la valoración del contenido en hexanal se presenta con un cierto interés práctico, no para controlar el nivel de oxidación lipídica sino lo correcto del mecanismo que lleva a través de la oxidación lipídica a unos componentes volátiles que pueden incidir en las cualidades organolépticas específicas del salchichón.

\section{BIBLIOGRAFIA}

Alford, J.A.; Smith, J.L. y Lilly, H.D.- "Relation of microbial activity to changes in lipids of foods"- J. Appl. Bact. 34 (1971), 133-146.

Astiasaran, I.; Villanueva, R. y Bello, J.- "Analysis of Proteolysis and Protein Insolubility during the Manufacture of Some Varieties of Dry Sausage".- Meat Science 28 (1990), 111-117.
Bailey, M.E.; Dupuy, M.P. y Legendre, M.G.- "Undesirable meat flavor and its control" in "The Analysis and Control of less desirable flavors in Foods and Beverages".- p. 31-52.

Charalambous, G.-(Ed.)-Academic Press, New York, 1980.

Chan, H.W.S.; Prescott, F.A. y Swoboda, P.A.T.- "Thermal decomposition of individual positional isomers of methyl linoleate hydroperoxide: evidence of carbon-oxygen bond scission".- J. Am. Oil Chemists Soc. 53 (1976), 572-576

De Ketelaere, A.; Demeyer, D.; Vandererckhove, P. y Vervaere, I."Stoichiometry of carbohydrate fermentation during dry sausage ripening".- J. Food Sci. 39 (1974), 297-300.

Dupuy, H.P.; Rayner, E.T. y Wadsworth, J.I.- "Correlations of flavor score with volatiles of vegetable oils".- J. Am. Oil Chemists'Soc. 53 (1976) 628-631.

Ellis, R.; Gaddis, A.M.; Currie, G.T. y Powell, S.L.- J. Am. Oil Chemists'Soc. 45 (1968), 553-559.

Frankel, E.N.; Neff, W.E. y Selke, E.- "Analysis of autoxidized fats by gas chromatography mass spectrometry. III Volatile thermal decomposition products of pure hidroperoxides from autoxidized and photosensitized oxidized methyl oleate, linoleate and linolenate".- Lipids 16 (1981), 279-285.

Gray, J.I. y Pearson, A.M.- Advances in Food Research, vol. 29, p. 1 86. - Chichester C.O., Mrak E.M. y Scheweigart E.S. (Ed.). 1984

Grosch, W.- "Autoxidation of unsaturated lipids".- cap. 4, p. 95-139.

Chan, H.W.S. (Ed.).- Academic Press, New York. (1987).

Henderson, S.R.; Witchwoot, A. y Nawar, W.W.- "The autoxidation of linoleates at elevated temperatures".- J. Am. Oil Chemists' Soc. 57 (1980), 409-413.

Henick, D.; Benca, A. y Mitchell, M.- "Estimating carbonyl compounds in rancid fats and foods".- J. Am. Oil Chemists'Soc. 31 (1954), 88-91.

Lizarraga, T.; Melgar, J. y Bello, J.- "Estudio de los cambios quimicos en los componentes grasos del chorizo con el proceso de curación".Grasas y Aceites 40 (1989), 370-375.

Loury, M.- "Posible mechanisms of autoxidative rancidity".- Lipids 7 (1972), 671-675.

McLeod, G. y Coppock, B.- "Volatile flavor components of beef boiled conventionally and microwave radiation".- Agric. Food Chem. 24 (1976), 835-843.

Melton, S.L.- "Methodology for following lipid oxidation in muscle foods".Food Technol. 37 (1983), 105-111

Morita, M. y Fujimari, M.J.- Agric. Food Chem. 21 (1973), 860-863.

Morita, M.; Tanara, M.; Tarayama, Y. y Yamamoto, Y.- "Metal-requising and non metal-reqiring catalysts in the autoxidation of methyl linoleate".- J. Am. Oil Chemists'Soc. 53 (1976), 487-488.

Persson, T. y Von Sydow, C.- "Aroma of canned beef. Gaschromatographic and mas-spectrometric analysis of the volatiles".- $\mathrm{J}$. Food Sci. 38 (1973), 377-385.

Shahidi, F.; Yun, J.; Rubin, L.J. y Wood, D.F.- "The hexanal content as an indicator of oxidative stability and flavor acceptability in cooked ground pork".- Can. Inst. Food Sci. Technol. J. 20 (1987a), 104-106.

Shahidi, F.; Rubin, L.J. y Wood, D.F.- Food. Chem., 23J. Food Sci. 53 (1987b), 151-157.

Shahidi, F.- "Flavor Chemistry", cap. 15, p. 188-201.- American Chemical Society, Washington DC, 1989

Tarladgis, B.G.; Watts, B.M.; Younathan, M.T. y Dugan, L.- "A destillation method for the determination of malonaldehyde in rancid foods".- $J$. Am. Oil Chemists' Soc. 37 (1960), 403-406.

UNE 55-023-73.- "Materias grasas. Indice de peróxidos".- AENOR. Ministerio de Industria. Madrid (1973).

UNE 55-118-79.- "Materias grasas. Determinación de la fracción de ácidos grasos de la grasa de mantequilla por cromatografia gaseosa".AENOR. Ministerio de Industria. Madrid (1979).

Warner, K.; Evans, C.D.; List, G.R.; Dupuy, H.P.; Wadsworth, J.I. y Goheen, G.E.- "Flavor score correlation with pentanal and hexanal contents of vegetable oil".- J. Am. Oil Chemists'Soc. 55 (1978) 252-256.

Yun, J.; Shahidi, F.; Rudin, L.J. y Diosady, L.L.- "Oxidative stability and flavor acceptability of nitrite-free meat-curing systems".- Can. Inst. Food Sci. Technol. J. 20 (1987) 246-251.

(Recibido: Octubre 1991) 